AG Winterstein PhD, College of Pharmacy, University of Florida, PO Box 100496, Gainesville, FL 32610. E-mail: almut@ufl.edu).

COMMENT. Spontaneous reports of adverse drug reactions to the FDA show a higher risk of cardiac events with amphetamines than methylphenidate. (FDA News;March 14, 2007). The above authors report a $20 \%$ increased risk for ED visits for cardiac symptoms for all stimulants combined (Winterstein AG et al. Pediatrics 2007;120(6):e1484). The present study did not confirm the previous report that amphetamines might carry a higher risk of adverse cardiac events than MPH. Further long-term population-based studies are indicated to define the risks of stimulant-induced serious heart events and the prophylactic utility of routine electrocardiograms before and during treatment.

\title{
DIFFUSION TENSOR IMAGING ABNORMALITIES IN THE CEREBELLUM OF CHILDREN WITH ADHD AND EPILEPSY/ADHD
}

Diffusion tensor imaging was used to investigate cerebellar structure in children with combined epilepsy/ADHD and ADHD alone, at the University of Basel, Switzerland. By generating fractional anisotropy (FA) maps, the extent to which water diffusion is greater in one direction compared with others, the organization of white matter in the brain is computed. Healthy controls $(n=12)$ exhibited more FA in the left and right middle cerebellar peduncle compared with 8 boys with combined epilepsy/ADHD, and more FA in the right middle cerebellar peduncle compared with 14 boys with developmental ADHD. Deficient cerebellar connections were demonstrated in both patient groups. Inattention and other ADHD problems in both epilepsy/ADHD and ADHD patients are based on the same neurobiological mechanisms that involve the middle cerebellar peduncle. (Bechtel N, Kobel $\mathrm{M}$, Penner I-K, et al. Decreased fractional anisotropy in the middle cerebellar peduncle in children with epilepsy and/or attention deficit/hyperactivity disorder: A preliminary study. Epilepsy \& Behav July 2009;15:294-298). (Respond: Dr Nina Bechtel, Dept Cognitive Psychology and Methodology, University of Basel, Missionsstrasse, 60/62, 4055 Basel, Switzerland. E-mail: nina.bechtel@unibas.ch).

COMMENT. One in 5 children with epilepsy has comorbid ADHD (Gross-Tsur et al, 1997). A study involving 203 patients found $60 \%$ of children with epilepsy had either ADHD-Inattentive subtype or ADHD-Combined. (Sherman EMS et al, 2007). Quality of life was impaired 2-fold in children with epilepsy complicated by ADHD-I, and 4-fold with ADHD-C comorbidity, when compared to normal controls. Impairment of attention is more likely with generalized epilepsies than with focal epilepsies, in most studies.

Approximately one in 4 children with ADHD has an abnormal EEG, without clinical seizures. The significance of subclinical seizure discharges in children with ADHD is controversial. In addition to EEG abnormalities, a neurobiological basis for ADHD is also demonstrated by MRI brain volume studies, PET studies, and neurological soft signs. MRI volumetric studies have found decreased volume of the total brain, right prefrontal cortex, cerebellar vermis, corpus callosum, and basal ganglia. (Castellanos FX et al. Arch Gen Psychiatry 1996;53:607-616). These developmental abnormalities correlate with frontostriatal-cerebellar circuit dysfunction, neuropsychological deficits, and response to stimulant 
medication. (Zametkin AJ, Rapoport JL. Neurobiology of ADHD. J Am Acad Child Adolesc Psychiatry 1987;26:676-686).

\section{PERIPHERAL NERVOUS SYSTEM DISORDERS}

\section{EARLY- AND LATE-ONSET INHERITED ERYTHROMELALGIA}

A genotype-phenotype relationship at the clinical, cellular and molecular levels is shown in a case of erythromelalgia of relatively late onset, in a study at Yale University School of Medicine, and centers in China. The patient, a male age 17 years, began experiencing excruciating pain, warmth and redness in both feet and lower legs at age 14 years. Blood was analyzed for mutations in SCN9A, gain-of-function sodium channel mutations that are preferentially expressed within dorsal root ganglia (DRG) and sympathetic ganglion neurons. The effect of the Q10R mutation on firing of DRG neurons was investigated by current-clamp recording. The hyperexcitability of the DRG neuron induced by Q10R mutation in this adolescent patient was smaller than the change produced by I848T, an early-onset erythromelalgia mutation. (Han C, Dib-Hajj SD, Lin Z et al. Early- and lateonset inherited erythromelalgia: genotype-phenotype correlation. Brain July 2009;132:17111722). (Respond: Stephen G Waxman, MD PhD, Department of Neurology, LCI 707, Yale University School of Medicine, 333 Cedar Street, New Haven, CT 06520. E-mail: Stephen.Waxman@yale.edu).

COMMENT. Inherited erythromelalgia (erythermalgia) (IEM) is an autosomal dominant disorder characterized by severe burning pain and erythema of the extremities triggered by warmth. IEM is linked to gain-of-function mutations in SCN9A, the gene encoding $\mathrm{Na}, 1.7$, a voltage-gated sodium channel that is preferentially expressed in dorsal root ganglion neurons, particularly nociceptors, and sympathetic ganglion neurons. The resultant nociceptor hyperexcitability causes pain in the extremities. Almost all cases reported are linked to families with onset in early childhood (infancy to 6 years of age). The above case report uncovers a new mutation in $\mathrm{Na}, 1.7, \mathrm{Q} 10 \mathrm{R}$, from a patient with onset in the second decade of life. Mutations that produce smaller effects on sodium channel activation are associated with a smaller degree of DRG neuron excitability and later onset of clinical signs.

Carbamazepine-responsive erythromelalgia and $\mathrm{Na}_{\mathrm{v}} 1.7$ mutation. The above team of investigators publish a second article on erythromelalgia (Fischer TZ et al. Ann Neurol July 2009;65:733-741), reporting a novel $\mathrm{Na}_{\mathrm{v}} 1.7$ mutation (V400M) in a threegeneration Canadian family with pain relieved by carbamazepine (CBZ). $\mathrm{Na}_{\mathrm{v}} 1.7$ sodium channels are preferentially expressed within nociceptor ganglia and sympathetic neurons that are involved in the inflammatory and neuropathic pain of inherited erythromelalgia. CBZ has a normalizing effect on mutant $\mathrm{Na}_{\mathrm{v}} 1.7$ channels in this Canadian kindred, preventing the hyperexcitability of dorsal root ganglia in erythromelalgia. 